\title{
Molecular Speciation and Antifungal Susceptibility Profile of Candida Species in a Tertiary Care Centre in Central Kerala
}

\author{
Reshma Bhaskaran ${ }^{1}$, Chithra Valsan², Sathiavathy K. A. ${ }^{3}$ \\ ${ }^{1}$ Department of Microbiology, Jubilee Mission Medical College and Research Institute, Thrissur, Kerala, India. \\ ${ }^{2}$ Department of Microbiology, Jubilee Mission Medical College and Research Institute, Thrissur, Kerala, India. \\ ${ }^{3}$ Department of Microbiology, Jubilee Mission Medical College and Research Institute, Thrissur, Kerala, India.
}

\section{ABSTRACT}

\section{BACKGROUND}

Candidiasis is a common life-threatening condition with various clinical manifestations. It can cause significant morbidity and mortality, especially in critically ill patients. Though mainly caused by $C$. albicans, it has shown a change in the epidemiological pattern with an increase in the prevalence of non albicans Candida (NAC) in the recent years. This change has also reflected in the profile of antifungal susceptibility since many of these species show high level of antifungal resistance associated with treatment failures. Thus, it is important to know the regional distribution of Candida species and also find their antifungal susceptibility profile to the commonly used antifungal agents.

\section{METHODS}

This is a descriptive study conducted for a period of two years on the clinical isolates of Candida species which satisfied the inclusion and exclusion criteria. Speciation was done by phenotypic methods which include germ tube test, chromogenic medium, corn meal agar and VITEK-2 system and by molecular methods using multiplex PCR. Antifungal susceptibility testing was done using automated method by VITEK-2 compact system.

\section{RESULTS}

Among the 80 isolates studied, on speciation $C$.albicans $25(31.25 \%)$ was the most common, followed by C. tropicalis 23 (28.75\%), C. parapsilosis 13 (16.25\%), C. krusei 12 (15\%), C. pelliculosa 4 (5\%), C. auris $2(2.5 \%)$ and C. glabrata $1(1.25 \%)$. Most isolates were susceptible to almost all the antifungal agents tested, but $C$. krusei showed high level of resistance.

\section{CONCLUSIONS}

C. albicans was the commonest individual species, but there was an overall predominance of non-albicans Candida (NAC). Speciation of Candida isolates is important as there is wide variation in their antifungal resistance pattern. Knowledge about the prevalent species and their antifungal susceptibility will help in early initiation of appropriate treatment, thus reducing the morbidity and mortality associated with Candida infections.

\section{KEY WORDS}

Candidiasis, Non Albicans Candida (NAC), Antifungal resistance, Candida Speciation

\author{
Corresponding Author: \\ Reshma Bhaskaran, \\ Department of Microbiology, \\ Jubilee Mission Medical College \& Research \\ Institute, Thrissur,P.0,-680005, \\ Kerala, India. \\ E-mail: scarletresh@gmail.com \\ DOI: $10.14260 /$ jemds $/ 2020 / 81$ \\ Financial or Other Competing Interests: \\ None. \\ How to Cite This Article: \\ Bhaskaran R, Valsan C, Sathiavathy KA. \\ Molecular speciation and antifungal \\ susceptibility profile of candida species in a \\ tertiary care centre in Central Kerala. J. \\ Evolution Med. Dent. Sci. 2020;9(06):357- \\ 362, DOI: $10.14260 /$ jemds $/ 2020 / 81$
}

Submission 09-12-2019,

Peer Review 18-01-2020,

Acceptance 24-01-2020,

Published 10-02-2020. 


\section{BACKGROUND}

Candidiasis is a common infection caused by yeast-like fungus, affecting both sexes, all age groups and is worldwide in distribution. Candidiasis includes diverse group of infections caused by Candida albicans or by other members of genus Candida. It produces infections that range from nonlife threatening mucocutaneous illness to invasive conditions which may involve virtually any organ. They are opportunistic pathogens in immunocompromised patients.[1]

There has been a significant increase in the number of reports of systemic and mucosal yeast infection over the last few years. These infections have also had a direct impact on the choice of empiric antifungal therapy and clinical outcome. Hence the clinical importance of species level identification and outcome has been recognized as the Candida species vary in the expression of virulence factors and antifungal susceptibility. Candida species are considered to be as the normal flora of human skin and mucosa but have been frequently reported as pathogens and the risk factors are due to excessive consumption of broad-spectrum antibiotics, underlying malignant diseases, HIV infections, organ transplantation, prolonged hospital stay and also exposure to invasive procedures.[2]

Species level identification of Candida species have become of utmost importance as many cases of Candida infections are being reported. For the past few years, an epidemiological shift has been noted from Candida albicans to non albicans Candida (NAC) species. As the conventional methods of identification of Candida species are often time consuming and may lead to inconclusive results, molecular diagnosis of Candidiasis has gained importance in the recent years because of its higher sensitivity and turn-around time.[3] The recent studies have suggested that with the introduction of fluconazole and itraconazole, there is increased prevalence of non albicans Candida (NAC) species. Infections with Candida tropicalis, Candida glabrata, Candida krusei and other Candida species are emerging as important opportunistic pathogens. This transition has a significant clinical impact due to the decreased susceptibility of many of these non albicans yeasts to antifungal agents. Thus, it is important to have an awareness about the different Candida species prevalent in a locality and their antifungal susceptibility pattern to start prompt and proper antifungal therapy thereby reducing the morbidity and mortality of such infections.

Mortality rates due to candidemia and disseminated candidiasis have not improved markedly over the last few years and have remained in the range of $30-40 \%$, corresponding to 2,800-11,200 deaths annually. One of the studies published in India in 2015 has shown that India has one of the highest rates of Candida bloodstream infections in the world. Mortality varied from $35-75 \%$ that is about 40,000 deaths among 14.3 million ICU patients in India each year.[4] Very few published data are available from Kerala on identification of Candida species. As the antifungal resistance is on a rise, we need to speciate the Candida species for proper treatment. In the present study, we aim to do identification of the Candida species both by conventional and molecular methods and also assessing the performance of the phenotypic methods for speciation of Candida species using molecular method as gold standard. In addition to that, we also aim to do antifungal susceptibility testing of the isolated Candida species. Therefore, early isolation, speciation and antifungal susceptibility will aid the clinicians to institute proper antifungal therapy thus decreasing morbidity and mortality.

\section{METHODS}

This descriptive study was performed in the microbiology department of a tertiary care hospital in Thrissur, Kerala during December 2017 to August 2019. Institutional ethics committee approval was obtained prior to the study. As per the suggestions from the statistician based on the prevalence observed in an earlier publication [5], a minimum of 76 Candida isolates had to be included in the present study. We included all clinically significant (as per the clinical history and microscopic picture of the samples) nonrepetitive isolates of Candida species, obtained from patient samples received in the routine microbiology laboratory, in the study. Isolates which appeared to be colonizers or commensals were omitted. The patient samples were sent by the treating doctors in appropriate containers and were cultured on routine media as per the laboratory SOP (standard operative procedures).

The isolates were subjected to speciation by conventional phenotypic methods which included Germ tube test and characteristics on Corn meal agar (Hi Media laboratories), colony colour on chromogenic medium (HiCrome agar by $\mathrm{Hi}$ Media laboratories), as well as by automated method by VITEK 2 compact system (Biomerieux Pvt Ltd) using ID-YST cards. All the instructions by the manufacturers were strictly followed during all the procedures. All isolates were also subjected to speciation by molecular method- Multiplex PCR (polymerase chain reaction) using the primers (Table. 1) and conditions given below. [6,7]

\section{DNA Isolation}

Fresh culture of the test isolates on Sabouraud's dextrose agar were used for colony PCR. A part of a single colony was picked with a sterile toothpick and suspended in $500 \mu \mathrm{l}$ sterile nuclease free water in PCR tubes, then subjected to boiling and freezing at $-80^{\circ} \mathrm{C}$, repeating this freeze thawing 3 to 4 times. PCR amplification: Multiplex PCR was carried out using the yeast specific primers and species-specific primers described in earlier studies. ${ }^{[4]}$ The amplification was performed in a 25 $\mu \mathrm{l}$ volume consisting of $2 \mathrm{x}$ PCR buffer, $0.6875 \mu \mathrm{l}$ of each primer, $10 \mu \mathrm{l}$ of DNA template and remaining volume consisting of sterilized water. PCR was carried out in a thermocycler under the following cycling conditions: 40 cycles of $15 \mathrm{~s}$ at $94^{\circ} \mathrm{C}$ denaturation, $30 \mathrm{~s}$ at $55^{\circ} \mathrm{C}$ annealing, and $45 \mathrm{~s}$ at $65^{\circ} \mathrm{C}$ extension, after a 10-min initial period of DNA denaturation and enzyme activation at $94^{\circ} \mathrm{C}$. The PCR products were analysed by electrophoresis with $2 \%$ agarose gels in TBE (trisborate- EDTA) buffer. The gels were stained with ethidium bromide $(75 \mu \mathrm{l}$ in $500 \mathrm{ml}$ distilled water) and PCR products were visualized with UV light using gel documentation system. Antifungal susceptibility testing: We also tested the susceptibility of these isolates to antifungal agents by automated VITEK 2 compact system using AST YS08 cards.

\section{Statistical Analysis}

On evaluation of the phenotypic methods with PCR as gold standard, the sensitivity and specificity of germ tube test (GTT), HiCrome Candida differential agar medium, corn meal 
agar (CMA) and VITEK-2 system was calculated to be $88 \%$ and $100 \% ; 91.25 \%$ and $100 \% ; 83.75 \%$ and $100 \%$; both $100 \%$ respectively.

\section{RESULTS}

A total of 80 clinically significant Candida isolates were enrolled in the study which satisfied the inclusion and exclusion criteria. Among the 80 isolates, 50 (62.5\%) isolates were from males and 30 (37.5\%) from females. Maximum number of samples belonged to the age group above 60 years (34) followed by neonates (23), 31-60 (17) and less than 30 years (6). The median age of study subjects was calculated to be 54 years. Thirty nine of these 80 Candida isolates were from blood samples, 19 from urine samples, 10 from pus and exudates and 12 from body fluids (table 2 ).

\section{Speciation of Isolates}

The phenotypic followed by molecular speciation (figure 1) of the isolates identified 25 isolates as $C$. albicans $(31.25 \%)$. The rest 55 isolates of nonalbicans Candida species included $23 \mathrm{C}$. tropicalis $(28.75 \%), 13$ C. parapsilosis $(16.25 \%), 12$ C. krusei (15\%), 4 C. pelliculosa (5\%), 2 C. auris $(2.5 \%)$ and one $C$. glabrata (1.25\%). Isolates of $C$. pelliculosa and $C$. auris were identified in the mycology section of All India Institute of Medical Sciences, New Delhi since primers for these species were not included in our study and hence could not be identified.

\section{Quality Control}

Quality control tests was carried out with standard ATCC strains of C. albicans (ATCC 10231) and C. tropicalis (ATCC 750 ) along with 8 bacterial strains and 2 fungal strains which were: E.coli, Klebsiella, Salmonella, Shigella, Pseudomonas, Staphylococcus, Streptococcus, Cryptococcus and Geotrichum species. None of the negative controls have shown any bands in PCR. The clinical specimens from which different Candida species were obtained are shown in the Table 3 . Among the 25 isolates of $C$. albicans, 7 were from pus samples, 5 from blood, 4 from urine and 9 from other body fluids. Non albicans Candida species were recovered from 34 blood samples, 15 urine samples, 3 pus and 3 other body fluids.

\begin{tabular}{|c|c|c|c|}
\hline $\begin{array}{c}\text { Candida } \\
\text { Species }\end{array}$ & $\begin{array}{c}\text { Primer } \\
\text { Name }\end{array}$ & Sequence (5'-3') & $\begin{array}{c}\text { Amplicon } \\
\text { Size (bp) }\end{array}$ \\
\hline Universal primers & UNI1 & GTCAAACTTGGTCATTTA & \\
& UNI2 & TTCTT TTCCTCCGCTTATTG & \\
\hline C. albicans & Calb & AGCTGCCGCCAGAGGTCTAA & $583 / 446$ \\
\hline C. glabrata & Cgla & TTGTCTGAGCTCGGAGAGAG & $929 / 839$ \\
\hline C. krusei & Ckru & CTGGCCGAGCGAACTAGACT & $590 / 169$ \\
\hline C. tropicalis & Ctro & GATTTGCTTAATTGCCCCAC & $583 / 507$ \\
\hline C. parapsilosis & Cpar & GTCAACCGATTATTTAATAG & $570 / 370$ \\
\hline C. guilliermondii & Cgui & TTGGCCTAGAGAT AGGTTGG & $668 / 512$ \\
\hline C. lusitaniae & Clus & TTCGGAGCAACGCCTAACCG & $433 / 329$ \\
\hline C. dubliniensis & Cdub & CTCAAACCCCTAGGGTTTGG & $591 / 217$ \\
\hline \multicolumn{4}{|r}{ Table 1. Primers Used for Identification of Candida Species } \\
\hline
\end{tabular}

Antifungal susceptibility profile (table 4): Antifungal susceptibility testing was done for all isolates except the two C. auris for which guidelines are not available. All the $C$. albicans strains were susceptible to the antifungal agents tested except one which was resistant to amphotericin B. $C$. tropicalis and C. pelliculosa showed $100 \%$ susceptibility to all the agents tested. $C$ krusei strains were $100 \%$ resistant to fluconazole, amphotericin B and caspofungin. All isolates were sensitive to micafungin. C. parapsilosis strains were $100 \%$ susceptible to fluconazole, amphotericin B, micafungin, caspofungin and flucytosine.

\begin{tabular}{|c|c|}
\hline Samples & Number (\%) \\
\hline Blood & $39(48.75 \%)$ \\
\hline Urine & $19(23.75 \%)$ \\
\hline Pus & $10(12.5 \%)$ \\
\hline Other body fluids & $12(15 \%)$ \\
\hline Total & $\mathbf{8 0}$ \\
\hline Table 2. Distribution of Samples \\
\hline
\end{tabular}

\begin{tabular}{|c|c|c|c|c|c|c|}
\hline \multicolumn{2}{|c|}{ Candida Species } & Total & Blood & Urine & Pus & $\begin{array}{c}\text { Other } \\
\text { Body } \\
\text { Fluids }\end{array}$ \\
\hline C. albicans (N=25) & & 25 & 5 & 4 & 7 & 9 \\
\hline \multirow{4}{*}{$\begin{array}{c}\text { Non albicans } \\
\text { Candida (NAC) } \\
\text { (N=55) }\end{array}$} & C. tropicalis & 23 & 7 & 13 & 2 & 1 \\
\cline { 2 - 8 } & C. parapsilosis & 13 & 10 & 1 & 1 & 1 \\
\cline { 2 - 8 } & C. krusei & 12 & 11 & 0 & 0 & 1 \\
\cline { 2 - 8 } & C. pelliculosa & 4 & 4 & 0 & 0 & 0 \\
\cline { 2 - 8 } & C. auris & 2 & 2 & 0 & 0 & 0 \\
\cline { 2 - 8 } & C. glabrata & 1 & 0 & 1 & 0 & 0 \\
\cline { 2 - 8 } & Total & $\mathbf{8 0}$ & $\mathbf{3 9}$ & $\mathbf{1 9}$ & $\mathbf{1 0}$ & $\mathbf{1 2}$ \\
\hline
\end{tabular}

Table 3. Distribution of Candida Species in Clinical Specimens

\begin{tabular}{|c|c|c|c|c|c|c|}
\hline $\begin{array}{c}\text { Candida } \\
\text { Species }\end{array}$ & FLC & VRC & AMP B & MICA. & 5FC & CASPO. \\
\hline $\begin{array}{l}\text { C. albicans } \\
(\mathrm{N}=25)\end{array}$ & $\begin{array}{c}25 \\
(100 \%)\end{array}$ & $\begin{array}{c}25 \\
(100 \%)\end{array}$ & $\begin{array}{c}24 \\
(96 \%)\end{array}$ & $\begin{array}{c}25 \\
(100 \%)\end{array}$ & $\begin{array}{c}25 \\
(100 \%) \\
\end{array}$ & $\begin{array}{c}25 \\
(100 \%) \\
\end{array}$ \\
\hline $\begin{array}{l}\text { C. tropicalis } \\
(\mathrm{N}=23)\end{array}$ & $\begin{array}{c}23 \\
(100 \%)\end{array}$ & $\begin{array}{c}23 \\
(100 \%) \\
\end{array}$ & $\begin{array}{c}23 \\
(100 \%) \\
\end{array}$ & $\begin{array}{c}23 \\
(100 \%) \\
\end{array}$ & $\begin{array}{c}23 \\
(100 \%) \\
\end{array}$ & $\begin{array}{c}23 \\
(100 \%) \\
\end{array}$ \\
\hline $\begin{array}{c}\text { C. krusei } \\
(\mathrm{N}=12)\end{array}$ & 0 & $\begin{array}{c}12 \\
(100 \%) \\
\end{array}$ & 0 & $\begin{array}{c}12 \\
(100 \%) \\
\end{array}$ & $\begin{array}{c}1 \\
(8.3 \%) \\
\end{array}$ & 0 \\
\hline $\begin{array}{c}\text { C. parapsilosis } \\
(\mathrm{N}=13)\end{array}$ & $13(100 \%)$ & $12(92.3 \%)$ & $13(100 \%)$ & $\begin{array}{c}13 \\
(100 \%)\end{array}$ & $\begin{array}{c}13 \\
(100 \%) \\
\end{array}$ & $\begin{array}{c}13 \\
(100 \%) \\
\end{array}$ \\
\hline $\begin{array}{l}\text { C. pelliculosa } \\
(\mathrm{N}=4)\end{array}$ & $\begin{array}{c}4 \\
(100 \%)\end{array}$ & $\begin{array}{c}4 \\
(100 \%) \\
\end{array}$ & $\begin{array}{c}4 \\
(100 \%)\end{array}$ & $\begin{array}{c}4 \\
(100 \%) \\
\end{array}$ & $\begin{array}{c}4 \\
(100 \%)\end{array}$ & $\begin{array}{c}4 \\
(100 \%) \\
\end{array}$ \\
\hline \begin{tabular}{|l} 
C. glabrata $(\mathrm{N}=1)$ \\
.
\end{tabular} & 0 & $1(100 \%)$ & $1(100 \%)$ & $1(100 \%)$ & $1(100 \%)$ & 0 \\
\hline Total $(\mathrm{N}=78)$ & $65(83.3 \%)$ & $77(98.7 \%)$ & $65(83.3 \%)$ & $78(100 \%)$ & $67(85.9 \%)$ & $65(83.3 \%)$ \\
\hline \multicolumn{7}{|c|}{ Table 4. Antifungal Susceptibility of the Candida Species } \\
\hline $\begin{array}{l}\text { *FLC- Fluconazo } \\
\text { 5FC- Flucytosin }\end{array}$ & VRC- & $\begin{array}{l}\text { onazole, } \\
\text { ofungin }\end{array}$ & & & & \\
\hline
\end{tabular}

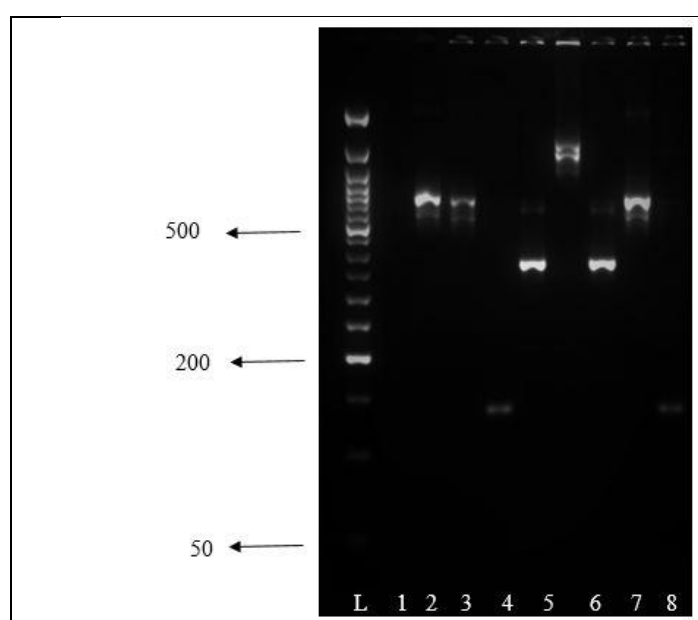

Figure 1. Agarose Gel Showing the Results Obtained for Multiplex PCR of Isolated Yeast Genomic DNA. Lanes: (L) 50-bp DNA Ladder, (1) Negative Control, (2) C. albicans, (3),(8) C. tropicalis, (4) C. krusei, (5),(7) C. parapsilosis, (6) C. glabrata

\section{DISCUSSION}

Candida infections have been increasingly reported as an important cause of mortality and morbidity in the recent years. Although $C$ albicans had been the predominant yeast causing such infections, an emergence of other species of Candida with increasing resistance to the commonly used antifungal agents have been increasingly recognized in the 
recent years globally. It is hence of utmost importance to have an awareness about the prevalent Candida species and their antifungal susceptibility pattern for prompt initiation of therapy and better clinical outcome.

The study was conducted to find out the distribution of various Candida species among the clinical isolates in our centre which is an 1800 bedded teaching hospital and their antifungal susceptibility pattern. A total of 80 Candida species isolated from clinical specimens that were received in the microbiology laboratory were enrolled in the study. The median age of the patients under study was 54 years. In the present study, males constituted $62.8 \%$ and females $37.1 \%$ of the patients. Among the 80 Candida isolates studied there were 25 (31.25\%) C. albicans and 55 (68.75\%) nonalbicans Candida species. The major non albicans species was $C$. tropicalis 23 (28.75\%) followed by C. parapsilosis 13 (16.25\%), C. krusei 12 (15\%), C. pelliculosa 4 (5\%), C. auris 2 (2.5\%) and C. glabrata $1(1.25 \%)$. Thus, in the present study though Candida albicans is still the commonest single isolate, overall, nonalbicans Candida species predominated.

Candida albicans had been the most commonly isolated species previously which accounts for $70-80 \%$ of the Candidiasis. During the last 10 years, this trend has been changing as NAC isolates are accounting for a major part of Candida infections. ${ }^{[7]}$ This change has been reported in many studies from India[ ${ }^{[8]}$ as well as abroad. ${ }^{[9,10]}$ Bhattacharjee $\mathrm{P}$ had reported Candida albicans as the commonest species (48.57\%) followed by Candida tropicalis (24.28\%).[8] In a study from North American medical centres, a predominance of nonalbicans species was noted; although $C$. albicans was the most frequently isolated species, it was followed by C. glabrata and other non- albicans Candida species.[11] Similar changes in the epidemiology have also been reported in studies from Latin American countries. In Chile, the prevalence of $C$. albicans has changed, with a progressive increase in non-albicans infections where $C$. parapsilosis was the most frequent species, followed by $C$. tropicalis and C. glabrata.[12,13] The reasons for this change in epidemiology could be severe immunosuppression, comorbidities, prematurity, prolonged antibiotic therapy, elderly patients, indwelling devices etc.

In our study, the commonest NAC species recovered was Candida tropicalis (28.75\%). This finding is consistent with many similar Indian studies also.[12] A study from Mumbai in 2019 has shown highest isolation rates for $C$. tropicalis (10/29) followed by C. parapsilosis ( 8/29), C. albicans (3/29) and $C$. krusei $(3 / 29)^{[14]}$. In a 3 years study conducted in trauma patients at All India Institute of Medical Sciences, $C$. tropicalis, $82(38.7 \%)$ was the most common isolated followed by $C$. parapsilosis, 43 (20.3\%) and C. albicans, 29 (13.7\%).[15] A surveillance study conducted in around 39 countries had shown increase in the prevalence of $C$. tropicalis (4.6\% in 1997 to $7.5 \%$ in 2003 ) and C. parapsilosis (4.2\% in 1997 to $7.3 \%$ in 2003 ) with a rise in isolation rates of rare species like $C$. guilliermondii, C. kefyr and C. rugosa. ${ }^{[16]}$

Candida krusei (15\%), Candida parapsilosis (16.25\%) and Candida tropicalis $(28.75 \%)$ are the predominant species from blood stream infections in our study. Candida parapsilosis has emerged as an important agent in nosocomial infections in several studies as reported in a review by Sardi et al.[17] In some other Indian studies like that of Singh et al.[18], Chakrabarti A et al.[19], Xess et al. [15], C. tropicalis is identified as the predominant species causing candidemia in North
India[15] and Maharashtra[14]. In contrast to these findings $C$. albicans still constitute the most prevalent fungal species in candidemia (46.3\%), followed by C. parapsilosis (19.5\%), C. glabrata $(15.9 \%)$, C. tropicalis $(14.6 \%)$ in a study from China by Xiao et al.[20]

In the present study we got 4 isolates of $C$. pelliculosa also which were isolated from an outbreak of nosocomial fungaemia in neonatal intensive care unit (NNICU). A study by Chakrabarti A. et al in 2001 highlights the importance of $C$. pelliculosa as an emerging pathogen in neonatal ICUs where he reports 379 cases ( $4.2 \%$ of admissions) over a period of 23 months.[21] During the study period we also got 2 isolates of Candida auris. Candida auris is an emerging multidrug resistant yeast which causes superficial and invasive infections with high mortality that has been reported globally and from India. The fungus $C$. auris was first noticed in India during a multicentric study of Candidemia across 27 ICUs in 2011.[22-24] It had caused infections in 5.3\% of these episodes, thus becoming fifth in position of the agents causing candidemia. ${ }^{23,24]}$

In our study, among the 19 Candida isolates from urine sample, $C$. tropicalis was the commonest (13/19) followed by C. albicans (4/19) and C. parapsilosis (1/19). A study conducted in Rajasthan in 2014 has shown the isolation rates of Candida in urine samples as $10.2 \%$. Here, the non albicans Candida group showed a predominance $(88.4 \%)$ over $C$. albicans (11.6\%). Among the non albicans isolates, most common was $C$. tropicalis, 61 (54.5\%) followed by C. glabrata $28(25 \%) .{ }^{[25]}$ In the present study all the isolates (except 2 isolates of $C$. auris) were tested for their antifungal susceptibility using automated method (VITEK 2 compact system) to Amphotericin B, Fluconazole, Voriconazole, Micafungin, Caspofungin and Flucytosine. All the 25 isolates of Candida albicans showed $100 \%$ susceptibility to all the abovementioned agents tested except one which was resistant to Amphotericin B. Highest level of resistance was shown by $C$. krusei. C. tropicalis, C. parapsilosis, C. pelliculosa and C. glabrata showed $100 \%$ invitro sensitivity to amphotericin B whereas $C$. krusei was $100 \%$ and C. albicans was $4 \%$ resistant to amphotericin B. Although amphotericin B has good activity against most species its use has been restricted due to the nephrotoxicity associated with it.

Fluconazole is the most common antifungal agent used for treatment of disseminated candidiasis and also for prophylaxis, especially in low birth weight infants. Excellent oral bioavailability also makes it the most preferred agent for treating fungal infections. In the present study fluconazole showed excellent in vitro activity against all $C$. albicans strains but resistant to all isolates of $C$. krusei and one isolate of $C$. glabrata. However, C. krusei are intrinsically resistant to fluconazole. ${ }^{[8]}$ Though some Indian studies have reported high rates of fluconazole resistance,[26] our findings show that it still can be continued as the first line agent in infections with $C$ albicans in our geographical area. In contrast, for infections with non albicans Candida it can be used only after testing the susceptibility. In a similar study, overall fluconazole resistance was reported as $34.8 \%$ in various species.[8] Overuse of fluconazole might be one among the reasons for the NAC predominance in the recent years. Voriconazole was sensitive in all strains except one $C$. parapsilosis strain. Voriconazole has high susceptibility rates in many of the earlier studies.[14] 
In our study, micafungin shows $100 \%$ sensitivity to all the 78 Candida isolates tested. Resistance to flucytosine and caspofungin was seen only for C. krusei. Caspofungin is reported to have good activity against many non albicans Candida and is now increasingly used for treatment of invasive infections especially health care associated infections with NAC. So, in our study, even though C. albicans is still the commonest individual species, there is overall predominance of NAC over $C$. albicans. Also, antifungal resistance was low as compared to other studies. It is important to know the prevalence of the Candida species in a geographical area which will help in proper management of the Candidiasis and also in the modification of antibiotic policies.

\section{CONCLUSIONS}

Candidiasis is recognized as an important cause of serious infections leading to high morbidity and mortality worldwide. The current changing epidemiological pattern and increasing incidence of non albicans Candida (NAC) have led to a hike in antifungal resistance pattern leading to failures in empirical therapy with the common antifungal agents. Knowledge about the prevalent Candida species in a geographical area will help the clinicians to institute proper antifungal therapy in appropriate dose thereby avoiding any treatment failures and mortality.

\section{ACKNOWLEDGEMENT}

I would like to express my sincere gratitude to Dr. Alex George of Jubilee Mission Medical College, Thrissur, and Dr. Immaculata Xess, Department of Mycology, All India Institute of Medical Sciences, New Delhi, for their technical support in carrying out PCR study.

\section{REFERENCES}

[1] Miceli MH, Díaz JA, Lee SA. Emerging opportunistic yeast infections. The Lancet infectious diseases 2011;11(2):142-51.

[2] Meurman JH, Siikala E, Richardson M, et al. Non-Candida albicans Candida yeasts of the oral cavity. Communicating Current Research and Educational Topics and Trends in Applied Microbiology 2007;1(1):719-31.

[3] Das P, Pandey P, Harishankar A, et al. A high yield DNA extraction method for medically important Candida species: a comparison of manual versus QIAcube-based automated system. Indian Journal of Medical Microbiology 2016;34(4):533-5.

[4] Kappy MS. Advances in Pediatrics, Vol. 2013, E-Book. Elsevier Health Sciences 2014 Nov 12.

[5] Khadka S, Sherchand JB, Pokhrel BM, et al. Isolation, speciation and antifungal susceptibility testing of Candida isolates from various clinical specimens at a tertiary care hospital, Nepal. BMC Research Notes 2017;10(1):218.

[6] Trost A, Graf B, Eucker J, et al. Identification of clinically relevant yeasts by PCR/RFLP. Journal of Microbiological Methods 2004;56(2):201-11.
[7] Carvalho A, Costa-De-Oliveira S, Martins ML, et al. Multiplex PCR identification of eight clinically relevant Candida species. Medical Mycology 2007;45(7):619-27.

[8] Bhattacharjee P. Epidemiology and antifungal susceptibility of Candida species in a tertiary care hospital, Kolkata, India. Current Medical Mycology 2016;2(2):20-7.

[9] Pfaller MA, Diekema DJ. Epidemiology of invasive candidiasis: a persistent public health problem. Clinical Microbiology Reviews 2007;20(1):133-63.

[10] Edmond MB, Wallace SE, McClish DK, et al. Nosocomial bloodstream infections in United States hospitals: a threeyear analysis. Clinical Infectious Diseases 1999;29(2):239-44.

[11] Horn DL, Neofytos D, Anaissie EJ, et al. Epidemiology and outcomes of Candidemia in 2019 patients: data from the prospective antifungal therapy alliance registry. Clinical Infectious Diseases 2009;48(12):1695-703.

[12] Ajenjo CMH, Aquevedo AS, Guzmán AMD, et al. Epidemiological profile of invasive candidiasis in intensive care units at a university hospital. Revista Chilena de Infectologia: Organo oficial de la Sociedad Chilena de Infectologia 2011;28(2):118-22.

[13] Jangla SM, Naidu R, Patel SC. Speciation and antifungal susceptibility testing of Candida isolates in various clinical samples in a tertiary care hospital in Mumbai. International Journal of Biomedical Research 2018;9(3):106-11.

[14] Gandham NR, Vyawahare CR, Jadhav SV, et al. Candidemia: speciation and antifungal susceptibility testing from a tertiary care hospital in Maharashtra, India. Medical Journal of Dr. DY Patil University 2016;9(5):5969.

[15] Tak V, Mathur P, Varghese P, et al. The epidemiological profile of candidemia at an Indian trauma care center. Journal of Laboratory Physicians 2014;6(2):96-101.

[16] Paul GL. ERG11 expression in azole resistant candida species isolated from diabetic patients in a tertiary care centre (doctoral dissertation, Tirunelveli Medical College, Tirunelveli) 2019.

[17] Sardi JCO, Scorzoni L, Bernardi T, et al. Candida species: current epidemiology, pathogenicity, biofilm formation, natural antifungal products and new therapeutic options. Journal of Medical Microbiology 2013;62(1):10-24.

[18] Singh R, Parija SC. Candida parapsilosis: an emerging fungal pathogen. The Indian Journal of Medical Research 2012;136(4):671-3.

[19] Chakrabarti A, Chatterjee SS, Rao KLN, et al. Recent experience with fungaemia: change in species distribution and azole resistance. Scandinavian Journal of Infectious Diseases 2009;41(4):275-84.

[20] Xiao Z, Wang Q, Zhu F, et al. Epidemiology, species distribution, antifungal susceptibility and mortality risk factors of Candidemia among critically ill patients: a retrospective study from 2011 to 2017 in a teaching hospital in China. Antimicrobial Resistance \& Infection Control 2019;8(1):89.

[21] Chakrabarti A, Singh K, Narang A, et al. Outbreak of Pichia anomala infection in the pediatric service of a tertiarycare center in Northern India. Journal of Clinical Microbiology 2001;39(5):1702-6. 
[22] Chowdhary A, Kumar VA, Sharma C, et al. Multidrugresistant endemic clonal strain of Candida Auris in India. European Journal of Clinical Microbiology \& Infectious Diseases 2014;33(6):919-26.

[23] Chakrabarti A, Sood P, Rudramurthy SM, et al. Incidence, characteristics and outcome of ICU-acquired Candidemia in India. Intensive Care Medicine 2015;41(2):285-95.

[24] Rudramurthy SM, Chakrabarti A, Paul RA, et al. Candida auris Candidaemia in Indian ICUs: analysis of risk factors. Journal of Antimicrobial Chemotherapy 2017; 72(6):1794-801.
[25] Singhal A, Sharma R, Meena VL, et al. Urinary Candida isolates from a tertiary care hospital: speciation and resistance patterns. Journal of The Academy of Clinical Microbiologists 2015;17(2):100-5.

[26] Adhikary R, Joshi S. Species distribution and anti-fungal susceptibility of Candidaemia at a multi super-specialty center in Southern India. Indian Journal of Medical Microbiology 2011;29(3):309-11. 